\title{
Size Effects on Nanowire Phonon Thermal Conductivity: a Numerical Investigation Using the Boltzmann Equation
}

\author{
D. Abouelaoualim \\ LPSCM, Department of Physics, Faculty of Sciences \\ Semlalia, Cadi Ayaad University \\ P.O. Box 2390, 40000 Marrakech, Morocco
}

(Received March 7, 2007)

\begin{abstract}
Analytical solution of the Boltzmann transport equation for phonon transport in $\mathrm{Bi}_{0.95} \mathrm{Sb}_{0.05}$ nanowire is obtained. Thermal conductivity was calculated from the analytical solution of the Boltzmann transport equation. We calculate the lattice thermal conductivity of $\mathrm{Bi}_{0.95} \mathrm{Sb}_{0.05}$ nanowire as a function of temperature for different wire thicknesses. The results show that thermal conductivity of nanowire can be significantly smaller than the bulk thermal conductivity. We show that low thermal conductivity $\mathrm{Bi}_{0.95} \mathrm{Sb}_{0.05}$ nanowire for thermoelectric applications would have a small diameter.
\end{abstract}

PACS numbers: 63.22.+m, 87.15.Aa

\section{Introduction}

Modern technology has enabled the fabrication of materials with characteristic dimensions of a few nanometers. Examples are superlattices [1-4], nanowires [5-8], and quantum dots [9-12]. Thermal transport in these low-dimensional nanostructures is important for next-generation microelectronic cooling techniques, novel solid-state energy conversion devices, and micro-nanoscale sensors. Thermal transport caused by lattice vibrations or phonons in nanostructures is very complicated due to the comparable phonon mean-free path, phonon wavelength, and characteristic size of nanostructures. The comparable length scales lead to novel transport phenomena that do not exist in bulk materials.

The possible enhancement of the thermoelectric figure of merit, $Z T=$ $T S^{2} \sigma / k_{\mathrm{e}}+k_{\mathrm{p}}$, where $S$ is the Seebeck coefficient, $\sigma$ is the electrical conductivity, $T$ is the temperature, and $k$ is the thermal conductivity for electrons (e) and phonons (p). Large values of $Z T$ require high $S$, high $\sigma$, and low $k$. Since an 
increase in $S$ normally implies a decrease in $\sigma$ because of carrier density considerations, and since an increase in $S$ implies an increase in the electronic contribution to $k$ as given by the Wiedemann-Franz law, it is very difficult to increase $Z T$ in typical thermoelectric materials. The best commercial 3D thermoelectric material is in the $\mathrm{Bi}_{2(1-2 x)} \mathrm{Sb}_{2 x} \mathrm{Te}_{3(1-2 y)} \mathrm{Se}_{3 y}$ family with room temperature $Z T \approx 1$ for $\mathrm{Bi}_{0.5} \mathrm{Sb}_{1.5} \mathrm{Te}_{3.3}$. Reduced dimensionality offers one strategy for increasing $Z T$ relative to bulk values. The use of low-dimensional systems for thermoelectric applications is of interest because low dimensionality provides: (1) a method for enhancing the density of states near $E_{\mathrm{F}}$, leading to an enhancement of the Seebeck coefficient, (2) opportunities to take advantage of the anisotropic Fermi surfaces in multi-valley cubic semiconductors, (3) opportunities to increase the boundary scattering of phonons at the barrier-well interfaces, without as large an increase in electron scattering at the interface, (4) opportunities for increased carrier mobilities at a given carrier concentration when quantum confinement conditions are satisfied, so that modulation doping and $\delta$-doping can be utilized. A significant progress has been made both theoretically and experimentally in understanding the thermal transport in nanostructures during last decade.

Several theoretical models have been established to calculate the thermal conductivity of nanowires. Molecular dynamics simulation [13], concept of net-radiation method of (phonon) radiative heat transfer to dispersive phonons [14] and equation of phonon radiative transport is rewritten to include anisotropic scattering by a particulate media by including an acoustic phase function and scattering term [15].

This paper intends to develop a theoretical modeling approach to investigate the size effect on in-plane thermoelectric transport of $\mathrm{Bi}_{1-x} \mathrm{Sb}_{x}$ nanowire using the phonon Boltzmann equation.

\section{Theory}

\subsection{Phonon dispersion and group velocities}

We calculate the acoustic phonon dispersion in free-standing cylindrical nanowires and thin films by solving the elasticity equation with given geometry and boundary conditions. We consider only the in-plane transport in the wire and the well and assume that the main contribution to the in-plane heat transfer comes from the longitudinal acoustic phonon modes. Since our main goal is comparison of the lattice thermal conductivity in thin films and nanowires, this assumption does not severely restrict the analysis. The dispersion relation for confined phonons in a nanowire of diameter $D$ is given by [16]:

$$
\begin{gathered}
\left(q^{2}-q_{\mathrm{t}}^{2}\right)^{2} \frac{\left(q_{\mathrm{d}} D / 2\right) J_{0}\left(q_{\mathrm{d}} D / 2\right)}{J_{1}\left(q_{\mathrm{d}} D / 2\right)}-2 q_{\mathrm{d}}^{2}\left(q^{2}+q_{\mathrm{t}}^{2}\right) \\
+4 q^{2} q_{\mathrm{d}}^{2} \frac{\left(q_{\mathrm{t}} D / 2\right) J_{0}\left(q_{\mathrm{t}} D / 2\right)}{J_{1}\left(q_{\mathrm{d}} D / 2\right)}=0,
\end{gathered}
$$

where $q$ is the in-plane component of the phonon wave vector, $J_{0}$ and $J_{1}$ are the 
ordinary Bessel functions, and $q_{\mathrm{d}}$ and $q_{\mathrm{t}}$ are two parameters given by

$$
q_{\mathrm{d}, \mathrm{t}}^{2}=\omega^{2} / v_{\mathrm{d}, \mathrm{t}}^{2}-q^{2} .
$$

Here $v_{\mathrm{d}}$ and $v_{\mathrm{t}}$ are the longitudinal and transverse sound velocities in the bulk material, respectively, and $\omega$ is the phonon frequency. Confined phonon dispersion is obtained by solving the above equations numerically, and phonon group velocity is calculated for each branch via numerical differentiation.

\subsection{Phonon Boltzmann equation}

A phonon of energy $\hbar \omega_{s}(q)$ and velocity $V_{s}(q)$ in the direction of $q$ gives the contribution of $\hbar \omega_{s}(q) V_{s}(q)$ to the heat current. The net phonon heat current with a small temperature gradient $T$ is given by

$$
J_{Q}=\sum_{q, s} N_{q, s} \hbar \omega_{s}(q) V_{s}(q)
$$

where subscript $s$ refers to a particular phonon polarization type, $q$ is the phonon wave vector, $\omega_{s}$ is the phonon frequency, $V_{s}(q)$ is the phonon group velocity, $\bar{N}_{q, s} \equiv N_{q, s}^{0}-N_{q, s}$ is the deviation of the phonon distribution, $N_{q, s}$, from its equilibrium value, $N_{q, s}^{0}$. The equilibrium phonon, $N_{q, s}^{0}$, is given by the BoseEinstein distribution

$$
N_{q, s}^{0}=\frac{1}{\exp \left(\hbar \omega_{s}(q) / k_{\mathrm{B}} T\right)-1} .
$$

By definition

$$
J_{Q}=k_{l} \nabla T \text {. }
$$

Thus, the problem of determining the lattice thermal conductivity is essentially that of obtaining $\bar{N}_{q, s}$. In order to do this we need to solve the Boltzmann equation for $\bar{N}_{q, s}$. In steady state, the phonon Boltzmann equation can be written as

$$
\left(\frac{\partial N_{q, s}}{\partial t}\right)_{\mathrm{drift}}+\left(\frac{\partial N_{q, s}}{\partial t}\right)_{\mathrm{scat}}=0
$$

In Eq. (6), $\left(\partial N_{q, s} / \partial t\right)_{\text {drift }}$ represents the change of the phonon distribution in the presence of a temperature gradient, and it is given by

$$
\left(\frac{\partial N_{q, s}}{\partial t}\right)_{\mathrm{drift}}=V_{s}(q) \nabla N_{q, s}=-\left(V_{s}(q) \nabla T\right) \frac{\partial N_{q, s}}{\partial t} .
$$

In the relaxation-time approximation $\left(\partial N_{q, s} / \partial t\right)_{\text {scat }}$ can be written as

$$
\left(\frac{\partial N_{q, s}}{\partial t}\right)_{\text {scat }}=\frac{N_{q, s}^{0}-N_{q, s}}{\tau_{\mathrm{C}}(q)}
$$

where $\tau_{\mathrm{C}}(q)$ is the combined phonon relaxation time in resistive processes. The combined phonon relaxation time is obtained from the Matthiessen rule [17]:

$$
\frac{1}{\tau_{\mathrm{C}}}=\frac{1}{\tau_{\mathrm{u}}}+\frac{1}{\tau_{\mathrm{M}}}+\frac{1}{\tau_{\mathrm{B}}}+\frac{1}{\tau_{\mathrm{ph}-\mathrm{e}}} .
$$

Here $\tau_{\mathrm{u}}$ is the relaxation time due to the anharmonic nature of the crystal potential energy (three-phonon Umklapp scattering); $\tau_{\mathrm{M}}$ is the relaxation time due to mass-difference scattering of phonons; $\tau_{\mathrm{B}}$ and $\tau_{\mathrm{ph}-\mathrm{e}}$ are relaxation times due 
to boundary and carrier scattering, respectively. The formulae for calculating $\tau_{\mathrm{u}}$, $\tau_{\mathrm{M}}, \tau_{\mathrm{B}}$, and $\tau_{\mathrm{ph}-\mathrm{e}}$ have been given in Ref. [18]. Substituting Eqs. (7) and (8) in Eq. (6) the phonon Boltzmann equation takes the form

$$
V_{x} \frac{\partial N_{q, s}}{\partial x}+V_{y} \frac{\partial N_{q, s}}{\partial y}+\frac{\bar{N}_{q, s}}{\tau_{\mathrm{C}}(q)}=V_{z} \frac{\partial T}{\partial z} \frac{\partial N_{q, s}^{0}}{\partial T},
$$

where $V_{x}, V_{y}$, and $V_{z}$ are the components of phonon group velocity along the $x$, $y$, and $z$ axis, respectively.

\subsection{Calculation of the lattice thermal conductivity}

The solution of Eq. (10) can be written as

$$
\bar{N}_{q, s}=\frac{\partial N^{0}}{\partial T} \frac{\partial T}{\partial z} V_{z} \tau_{\mathrm{C}}
$$

Substituting Eqs. (4) and (11) in Eq. (3) and comparing the result with Eq. (5), we obtain the formula for the lattice thermal conductivity

$$
k_{l}=\left(\frac{k_{\mathrm{B}}}{\hbar}\right)^{3} \frac{k_{\mathrm{B}}}{2 \pi^{2} V} T^{3} \int_{0}^{\theta_{\mathrm{D}} / T} \frac{\tau_{\mathrm{C}} x^{4} \mathrm{e}^{x}}{\left(\mathrm{e}^{x}-1\right)^{2}} \mathrm{~d} x .
$$

\section{Results of computations and discussion}

Figure 1 shows the dispersion relations of the lowest confined acoustic phonon branches in a $\mathrm{Bi}_{0.95} \mathrm{Sb}_{0.05}$ cylindrical nanowire with a diameter of $15 \mathrm{~nm}$. We can see that the branch has a linear dispersion relation for very small values of $q$. The group velocity for the branch almost coincides with the bulk velocity [19] for very small values of the phonon energy.

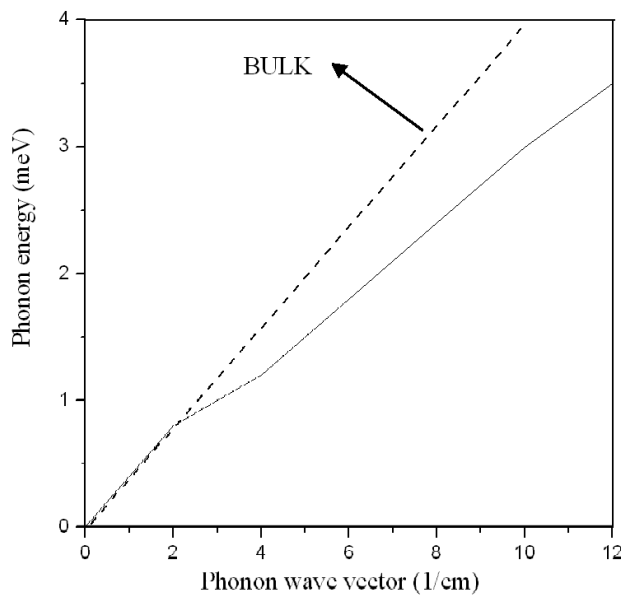

Fig. 1. Acoustic phonon dispersion relation for confined branches in a cylindrical nanowire with a diameter of $15 \mathrm{~nm}$.

After the group velocity is found, we calculate the lattice thermal conductivity. Figure 2 is a plot of the thermal conductivity of $\mathrm{Bi}_{0.95} \mathrm{Sb}_{0.05}$ cylindrical 
nanowire versus temperature. At low temperatures the phonon wavelength is long and if the phonon wavelength is larger than the size of defects, there will be a little scattering from defects. Additionally, long wavelengths correspond to small

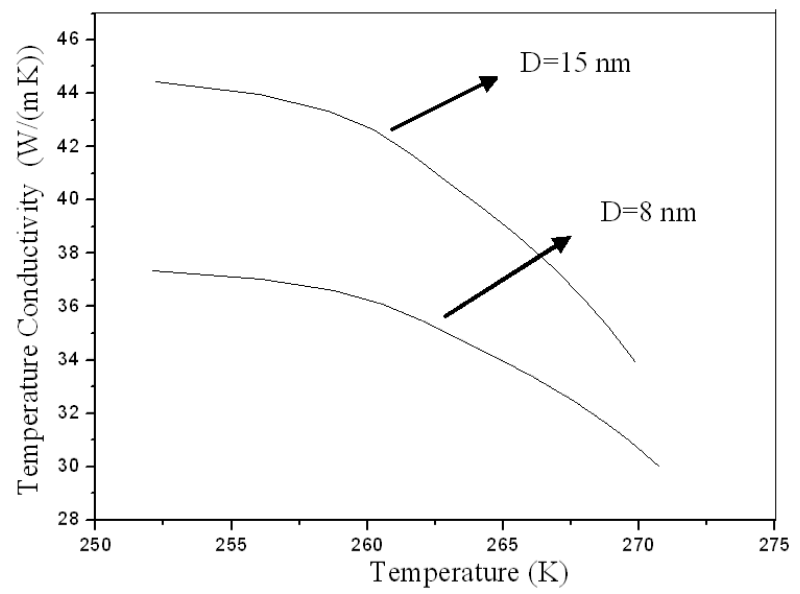

Fig. 2. Lattice thermal conductivity as a function of temperature for a $\mathrm{Bi}_{0.95} \mathrm{Sb}_{0.05}$ nanowire of diameter $D=10 \mathrm{~nm}$ and $D=15 \mathrm{~nm}$.

wave vectors so the Umklapp scattering is unlikely to happen. Therefore, at low temperature the dominant scattering mechanism is boundary scattering, and the measured thermal conductivity may depend on the size of the sample. However, since the mean free path is determined by the boundary scattering as a fixed value, the thermal conductivity is proportional to the specific heat and shows $T^{3}$ temperature dependence at very low temperature. As the temperature increases, the phonon wavelength decreases and becomes close to the size of the defects and defect scattering starts to dominate the phonon transport. For even a higher temperature, the phonon wavelength continues to decreases and eventually the wave vectors get large enough and the Umklapp scattering becomes the dominant scattering mechanism. For alloys or materials with a large number of defects, defect scattering may dominate over most of the temperature range. When diameter is smaller $(D=8 \mathrm{~nm})$ the effective thermal conductivity is reduced significantly.

\section{Conclusions}

In this paper, we presented a theoretical model for calculating the lattice thermal conductivity in $\mathrm{Bi}_{0.95} \mathrm{Sb}_{0.05}$ nanowire. Phonon Boltzmann equation is employed to establish a lattice thermal conductivity. The results show that thermal conductivity of $\mathrm{Bi}_{0.95} \mathrm{Sb}_{0.05}$ nanowire can be significantly smaller than the bulk thermal conductivity. We show that low thermal conductivity nanowire for thermoelectric applications would have a small diameter. 


\section{References}

[1] L. Esaki, IEEE J. Quant. Electron. QE-22, 1611 (1986).

[2] E.E. Mendez, F. Agullo-Rueda, J.M. Hong, Phys. Rev. Lett. 60, 2426 (1988).

[3] D. Abouelaoualim, Semicond. Phys. Quantum Electron Opto. 7, 333 (2004).

[4] D. Abouelaoualim, Prama. J. Phys. 66, 455 (2006).

[5] S.G. Volz, G. Chen, Appl. Phys. Lett. 75, 2056 (1999).

[6] S.G. Volz, D. Lemonnier, J. Saulnier, Microscale Thermophys. Eng. 5, 191 (2001).

[7] Z.L. Wang, R.P. Gao, Z.W. Pan, Z.R. Dai, Adv. Eng. Mater. 3, 657 (2001).

[8] Y. Wu, R. Fan, P. Yang, Nano Lett. 2, 83 (2002).

[9] M.A. Kastner, Physics Today 46, 24 (1993).

[10] L. Jacak, P. Hawrylak, A. Wojs, Quantum Dots, Springer, Berlin 1998.

[11] D. Pfannkuche, V. Gudmundsson, P.A. Maksym, Phys. Rev. B 47, 2244 (1993).

[12] N.A. Bruce, P.A. Maksym, Phys. Rev. B 61, 4718 (2000).

[13] A.A. Kiselev, K.W. Kim, M.A. Stroscio, Phys. Rev. B 62, 6896 (2000).

[14] C. Dames, G. Chen, J. Appl. Phys. 95, 682 (2004).

[15] A. Balandin, K.L. Wang, Phys. Rev. B 58, 1544 (1998).

[16] Se Gi Yu, K.W. Kim, M.A. Stroscio, G.J. Iafrate, Phys. Rev. B 51, 4695 (1995).

[17] A. Balandin, K.L. Wang, Phys. Rev. B 58, 1544 (1998).

[18] J. Zou, A. Balandin, J. Appl. Phys. 89, 2932 (2001).

[19] M.S. Dresselhaus, T. Koga, X. Sun, S.B. Cronin, K.L. Wang, G. Chen, in: 16th Int. Conf. on Thermoelectrics: Proc., ICT'97, Dresden 1997, Eds. A. Heinrich, J. Schumann, IEEE, Piscataway, N.J. 1997, p. 12. 